\title{
On investigating the flow through an axisymmetric channel with sudden changes in geometry
}

\author{
C. Giurgea ${ }^{1}$, F. Bode ${ }^{1, a}$, L. Nascutiu ${ }^{1}$, D. Banyai ${ }^{1}$, L. Marcu $^{1}$ \\ ${ }^{1}$ Technical University of Cluj Napoca, Faculty of Mechanics, Dept. of Mechanical Engineering, 400103, Romania
}

\begin{abstract}
The paper presents the authors first experience in the complementary use of the CFD and PIV methods for the investigation of a steady flow of an incompressible fluid through a successive sudden contraction (diameters ratio 4:1) and expansion (diameters ratio 1:2). The investigation of this flow was chosen by the authors as a test case for an assessment of the capability of a standard PIV system to make accurate measurements of the $2 \mathrm{D}$ velocity field. With this aim in view, in a first step of the study the flow was investigated by numerical simulations using the CFD code FLUENT, in order to map the velocity field and to identify the zones covered by recirculation flow. An experimental set-up was built and PIV investigations were conducted for validating the numerical simulation results. It was observed that there currently exist important technical limitations of the experimental setup in assessing the velocities close to the walls, and implicitly affecting the possibility of validating the results of the numerical simulation. That is why, based on the PIV investigation, at the current stage of the study the authors can only advance qualitative observations and identify possible solutions to upgrade the PIV setup.
\end{abstract}

\section{Introduction}

In biomedical applications concerning the blood flow through stenoses, bifurcations or vascular prostheses. there are several flow features of particular interest, such as velocity profiles, recirculation zone/vortex occurrence and evolutions, as well as wall shear stress distributions. These features are very difficult to measure in vivo but over the last ten years there have been published many studies that aimed to evaluate them by using CFD (Computational Fluid Dynamics) based numerical simulations [1].

In order to validate the numerical results, investigations based on PIV (Particle Image Velocimetry) methods were undertaken [2]. A condition to assess the flow features by experimental investigations based on the PIV method is having the capability to accurately measure the velocities close to the walls. Despite numerous recent experiments, the assessment of the near wall velocity field is still a challenge for researchers working on this subject [3].

PIV is an optical non-intrusive velocity measurement method that could be briefly described as follows. A plane in the flow field seeded with light scatter particles has to be illuminated with two successive laser pulses delayed by a time $\Delta \mathrm{t}$. The so-

\footnotetext{
a florin.bode@termo.utcluj.ro
}

called "frame-straddling" refers to positioning the two laser pulses on sequential CCD frames in such a manner that the positions of particles at the two instants of time are recorded by a CCD camera on different frames. The captured images are divided in interrogation areas (usually having the size of $32 \times 32$ or $64 \times 64$ pixels) and the displacement of the particles within each pair of corresponding interrogation areas that belong to two successive images is calculated by applying a cross-correlation algorithm. Knowing $\Delta t$ and the particles displacement, the average velocity of particles within an interrogation area is obtained.

The accurate near wall measurements of the velocity field by using PIV could be impeded by: image distortions, unfocused image, sparse particle distribution or even lack of particles in the region close to the wall. In order to avoid these difficulties, the standard PIV technique works with a reduced optical magnification degree of the camera recorded image less DOF (Depth of Field) but larger field of view. Processing this type of images results in information about a larger flow field, but the chances to assess the velocity distribution near the wall can be seriously diminished.

With a view towards a forthcoming research project focused on the experimental investigations of a flow through a femoral artery stenosis and bypass, the authors had to start with checking the capability of a 
standard 2D PIV set-up to make accurate measurements in channels with small diameters and variable geometry and, if necessary, to identify solutions to upgrade the technique already implemented in their laboratory.

The flow through sudden expansions/contractions is considered a "classical problem" of fluid mechanics [4]. The complexity of this separating and reattaching flow made it a challenging research subject. Over the last twenty years, the flow through sudden expansion/contraction has become a standard problem to test the performances of different numerical methods and to validate their results by experiments [5].

In respect to this and to the authors' objective, the investigation of a steady flow of an incompressible fluid through a successive sudden contraction (diameters ratio $4: 1$ ) and expansion (diameters ratio $1: 2$ ) was chosen by the authors as a test case.

In a first step of the study, numerical simulation of this type of flow was undertaken using the CFD software FLUENT in order to obtain the velocity field and to identify the flow structures. The numerical simulation results will be the baseline for the PIV investigations.

\section{Methods and materials}

Considering that the diameters and the geometry of the channel to be investigated by PIV had to be as similar as possible to those of a femoral artery stenosis (6-8 $\mathrm{mm}$ diameter) the authors had to find a clear model with an adequate geometry. Thus, the successive sudden contraction and expansion profile of the channel resulted from extracting the adjustable throttle screw from a clear phantom model of a flow control valve with one way adjustment (model D: TM-H-1GRO, FestoDidactic GmBh, figure 1) and chocking the second inlet of the valve (figure 2).

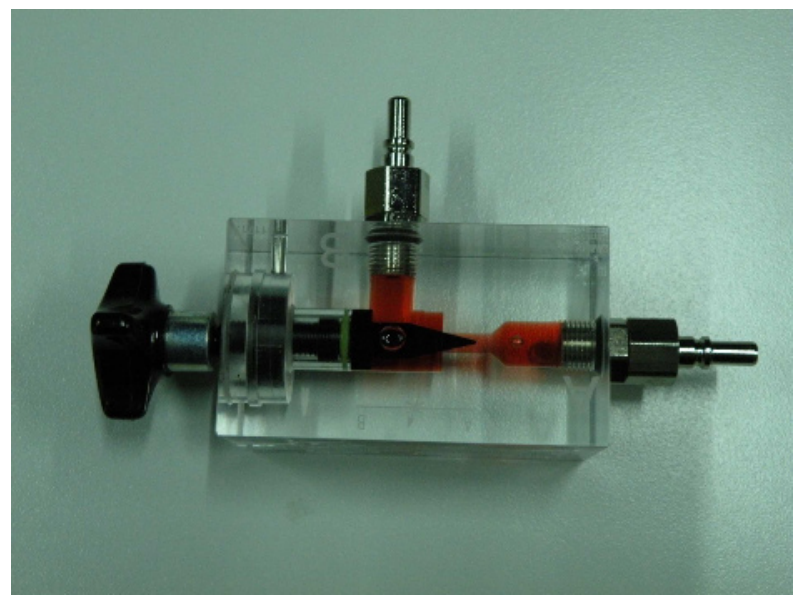

Fig. 1. The clear phantom model of a flow control valve with one way adjustment

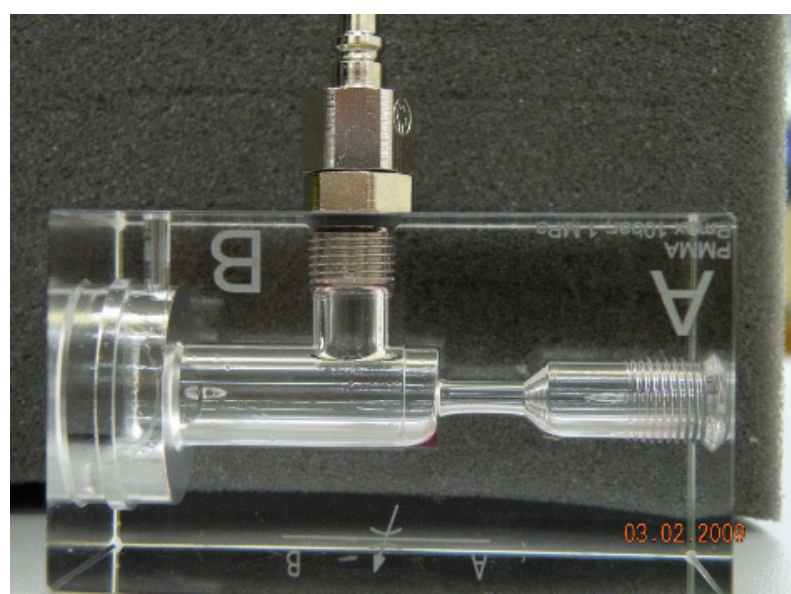

Fig. 2. The clear model of the axisymmetric channel

\subsection{CFD INVESTIGATION}

The geometry of the computational domain was established in accordance with the axisymmetric profile of the channel presented in figure 2 . Thus, a 3 $\mathrm{mm}$ diameter and $7.5 \mathrm{~mm}$ long throat connects the 12 $\mathrm{mm}$ diameter and $36 \mathrm{~mm}$ long upstream part of the channel to the $6 \mathrm{~mm}$ diameter and $14 \mathrm{~mm} \mathrm{long}$ downstream part of the channel, thus forming the successive sudden contraction (diameters ratio 4:1) and expansion (diameters ratio 1:2) as can be seen in figure 2 .

The geometrical model was made in Ansys Design Modeler, and the configuration is a $3 \mathrm{D}$ one. The geometrical discretization was realized in Ansys ICEM CFD and consists of tetrahedral elements, the total number of cells being 443000 (figure 3 ).

The studies were performed with the help of the well known CFD software FLUENT. A segregated solver formulation was used for these computations (equations are solved sequentially instead of simultaneously as in coupled solver).

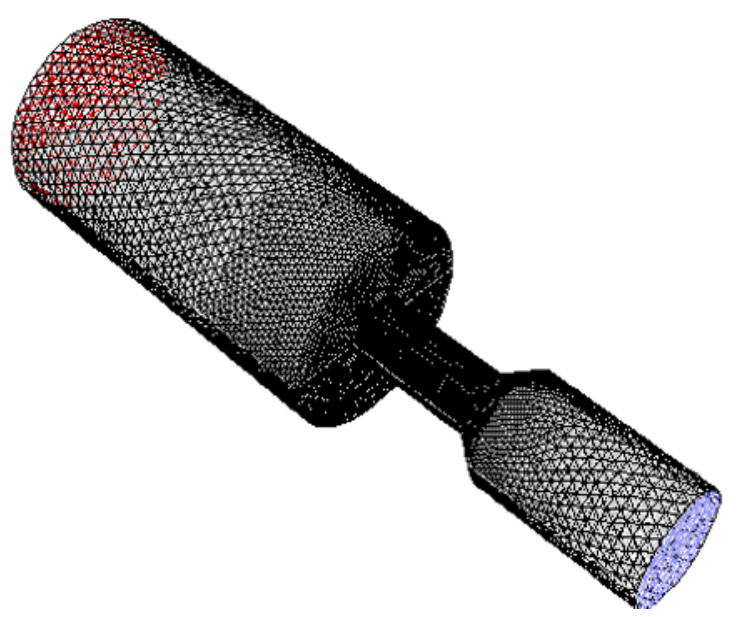

Fig. 3. Geometrical discretization of the studied domain 
Regarding the accuracy of the results and the convergence criterion, we have imposed a $10^{-4}$ convergence criteria for all the variables residuals. The viscous model used for this study is RNG $k-\varepsilon$. The SIMPLE algorithm is used for pressure-velocity coupling.

A constant velocity profile was imposed at the inlet. The mean velocity value of $0.3 \mathrm{~m} / \mathrm{s}$ was established in accordance with the minimum flow rate (2.4 $1 / \mathrm{min}$ ) provided by the centrifugal pump used to generate the flow in the experimental set-up. The Reynolds number calculated based on this values and the diameter of the upstream part of the channel was $\mathrm{Re}=3600$.

\subsection{PIV EXPERIMENTAL INVESTIGATION}

The experimental setup used for the PIV investigations is shown in figure 4. It can be divided in two principal areas: the hydraulic closed-loop and the 2D PIV system.

In the hydraulic closed-loop the steady flow is generated by an inverter driven variable speed centrifugal pump (model F127, Armfield, UK). During the experiments the pump speed was set at $600 \mathrm{rpm}$, which corresponds to a flow rate of $2.4 \mathrm{l} / \mathrm{min}$. Hoses with quick release fittings were used for easy connection of the pump with the water reservoir and the testing zone.

The detail in figure 5 presents the clear acrylic glass test section having an internal axisymmetric channel that is similar in geometry to that used as a flow domain model in the CFD investigation.

The working fluid was water. To conduct the optical investigation, the water was seeded with $10 \mu \mathrm{m}$ diameter hollow glass spheres as light scatters. The ratio between the refractive index of the acrylic glass and that of the water is 1.119 [6].

The flow in the horizontal plane passing through the test section centreline was investigated using the TSI 2D PIV System (TSI Incorporated, USA).

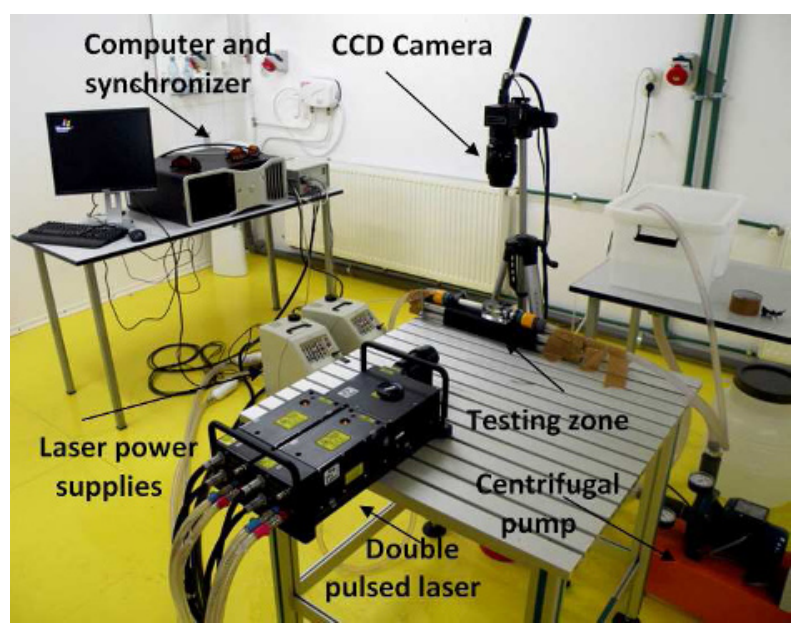

Fig. 4. The experimental set-up used for the PIV investigation

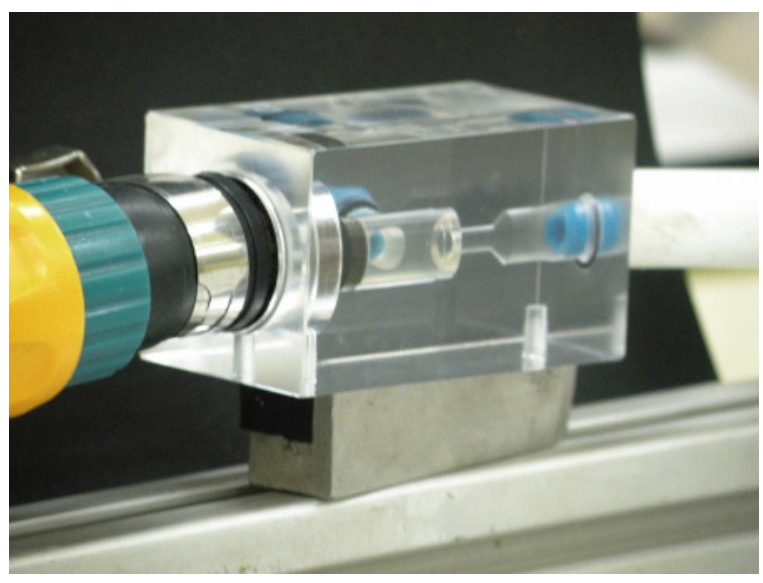

Fig. 5. Detail of the testing zone

The illumination of the flow field is assured by a double pulse laser head Nd:Yag that provides two 532 $\mathrm{nm}$ green light pulses, each one with a duration of 10 $\mathrm{ns}$ and an energy of $120 \mathrm{~mJ}$. Each laser beam is expanded into a luminous sheet with a $1 \mathrm{~mm}$ thick waist by means of the lightsheet optics. In the experiments presented here, the light sheet optics consisted of a $500 \mathrm{~mm}$ focal length spherical lens and a -25 focal length cylindrical lens.

The firing frequency of each laser is $15 \mathrm{~Hz}$ but the cross-pulse delay is adjustable and externally controlled by the Laser Pulse Synchronizer that provides all the control and activation signals including those for precision frame straddling. The syncronizer is programmed through the INSIGHT $3 \mathrm{G}$ software running on a Dell Workstation (Intel Dual Core Xenon 5150).

The images of the particles are recorded with the Powerview 17 CCD camera that has: 4 MP $(2048 \times 2048$ pixels $)$ resolution, an image capture rate of 16 frames/second and the ability to record sequential images in separate frames. The camera is placed above the model and perpendicular to the flow field. In the standard configuration of our PIV system the camera is equipped with the Nikon AF Nikkor 50 $\mathrm{mm} \mathrm{f} / 1.8 \mathrm{D}$ lens (Nikon, Japan).

The ensemble PIV algorithm, the RecursiveNyquistGrid, FFT correlator and interrogations windows of $64 \times 64$ were used to process the images and obtain the velocities' vectors.

\section{Results and discussions}

The results obtained by numerical simulation are presented in figures 6-10. The contours of the streamwise velocity in figure 6 , the velocity lines in figure 7 , the isocontour for zero streamwise velocity in figure 8 as well as the streamwise velocity profiles in figure 9 clearly show the axial symmetry of the flow throughout the investigated flow domain. This observation is interesting in that, based on his experimental results, Durst, recently cited by Dalal [7], justified the appearance of assymetric flow with increasing Reynolds number even in symmetrical channel with both right angle or $45^{\circ}$ expansion. 


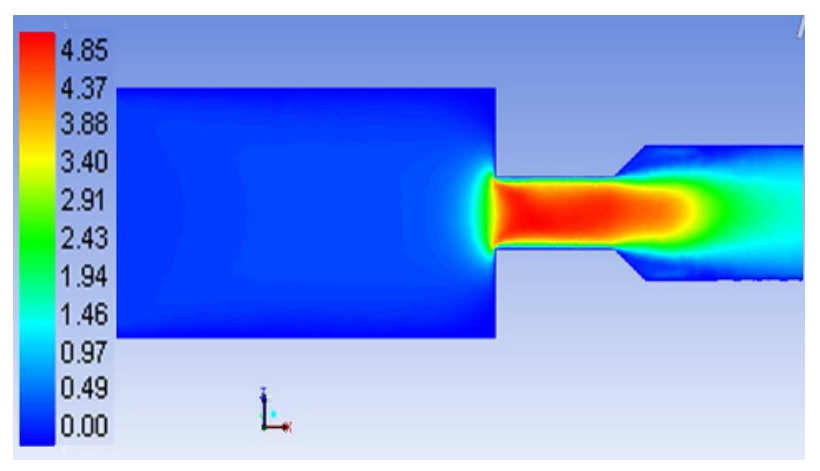

Fig. 6. Contours of streamwise velocity

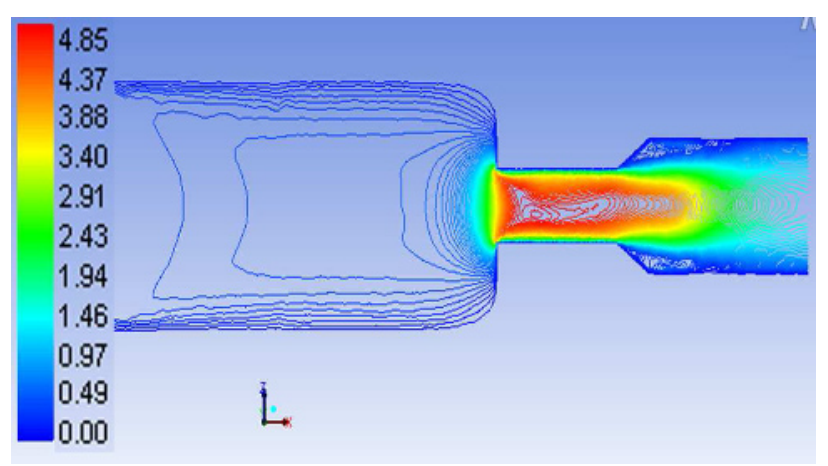

Fig. 7. Streamwise velocity lines

As it was mentioned in [7], generally the flow through a sudden contraction develops recirculation zones immediately after the entrance in the constricted axisymmetric channel for a relatively large Reynolds number. Even though in this case the Reynolds number based on the throat diameter and the mean velocity of $3.8 \mathrm{~m} / \mathrm{s}$ is $\operatorname{Re}=45000$, the appearance of a recirculation zone at the throat entrance could not be identified in figure 8 .

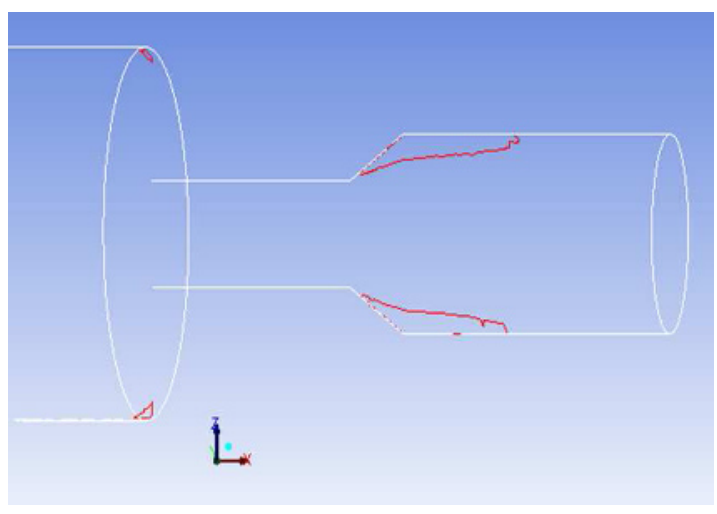

Fig. 8. Isocontour for zero streamwise velocity

The isocontour of zero streamwise velocity delineates the symmetrical recirculation zones (figure 8).

Furthermore, by observing the streamwise velocity profiles in figure 9 one can notice that along the throat the velocity profile is almost the same and is characteristic of a turbulent flow. The separation of the boundary layer just at the exit of the throat, at the point where the streamwise velocity becomes negative, the formation of the recirculation zone where the streamwise velocity remains negative and the reatachment point on the downstream part of the channel, where the streamwise velocity becomes again positive at the wall, are evident in figure 9.

Both symmetrical recirculation zones formed by the flow at the sharp edge of the upstream part of the channel (contraction exit) and at the throat exit (expansion entrance) are shown in figure 8 and in details in figure 10.

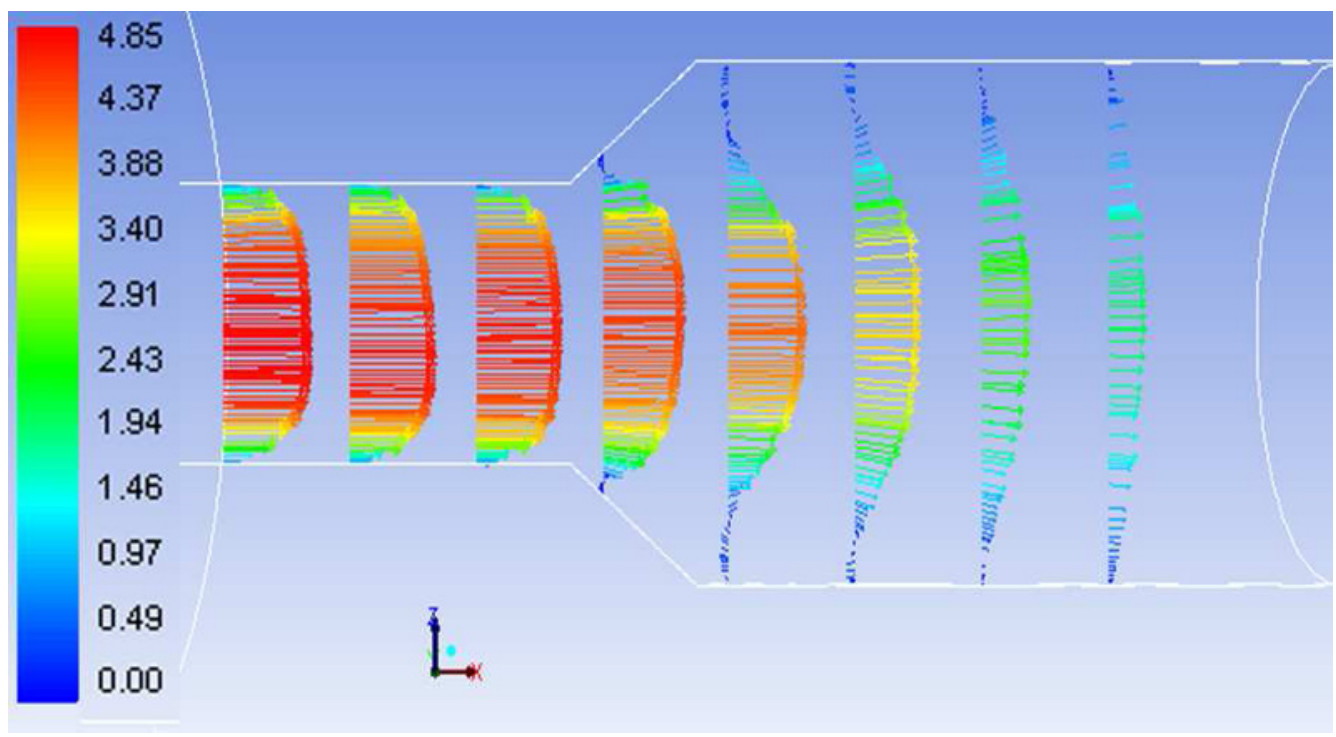

Fig. 9. Streamwise velocity profiles 
Despite numerous studies concerning the flow through sudden contraction/expansion, it is difficult to compare our results with those already reported. One of the reasons is that the majority of these studies investigate the flow either through sudden contraction or through sudden expansion, the channels having different configurations and only few of those beeing circular in section. The flow through axisymmetric successive sudden contraction/expansion is analysed mostly in the papers that referred to the flow through a stenosis artery, but in that case the flow is laminar. As it was highlighted in [7], [8], [9] the channel geometry, the contraction and expansion ratio, the sharpness of the throat in the channel, the velocity profile imposed at the inlet of the expansion as well as the Reynolds number could influence the symmetry or the asymmetry of the flow and, most importantly and relevantly for the biomedical application, the occurrence, the location and the strength of the recirculation zone. Taking this into account, and the fact we had restricted our study for only one Reynolds number and a single imposed profile of entry velocity, the two observations that are not entirely concordant with the results of other studies must be regarded prudently until other numerical simulations are done for further cases.

The symmetry of the flow in the expansion zone and the presence of a recirculation zone with the same location as identified in the results of the numerical simulation was evidenced also in the results of the PIV flow investigation. Figure 11 shows the image of the seeded flow field through the expansion entrance, on which we superimposed the velocity field obtained through the analysis of images and the PIV data processing.
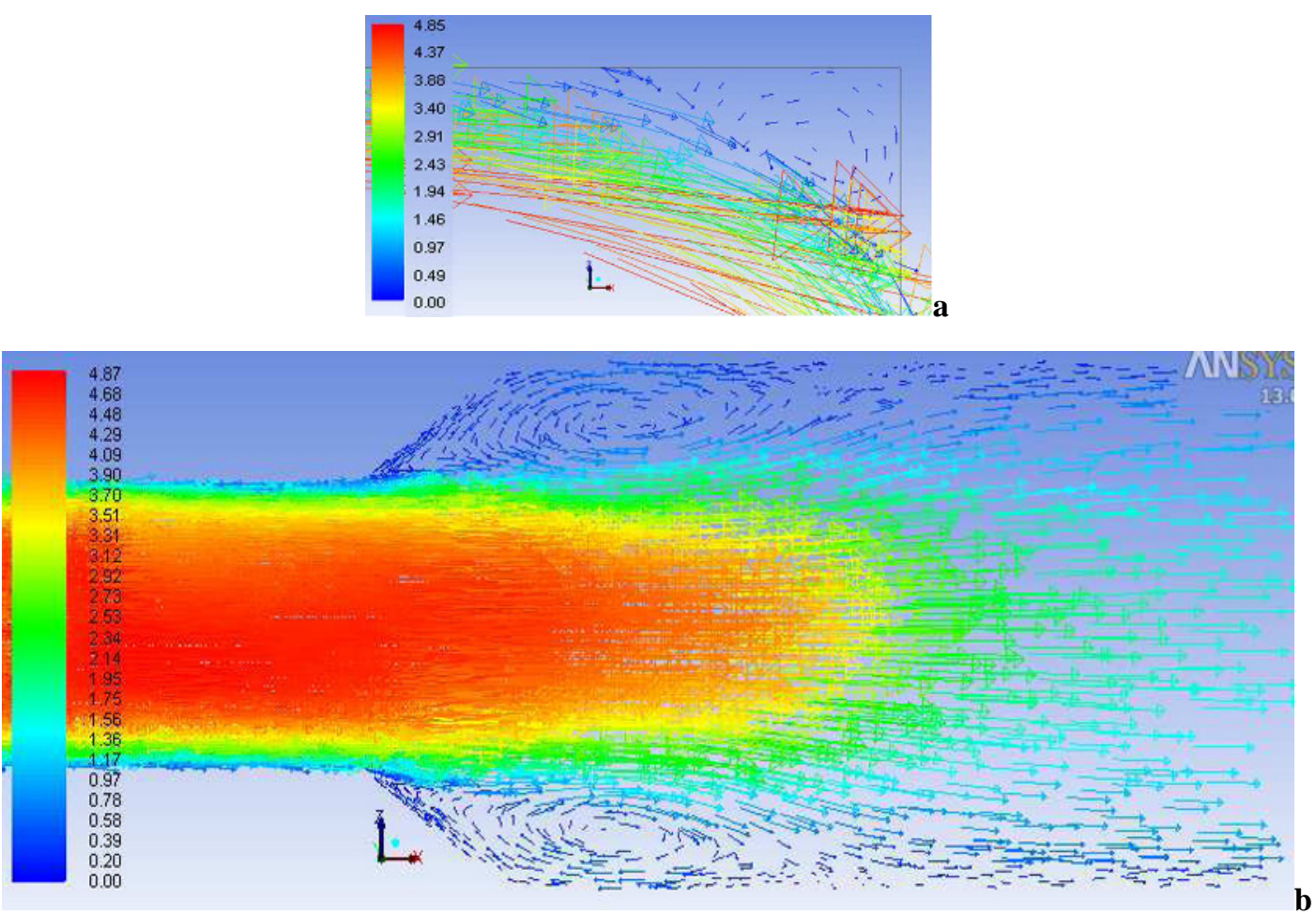

Fig. 10. Details of the recirculation zones on the sharp edge (a) and at the expansion (b)

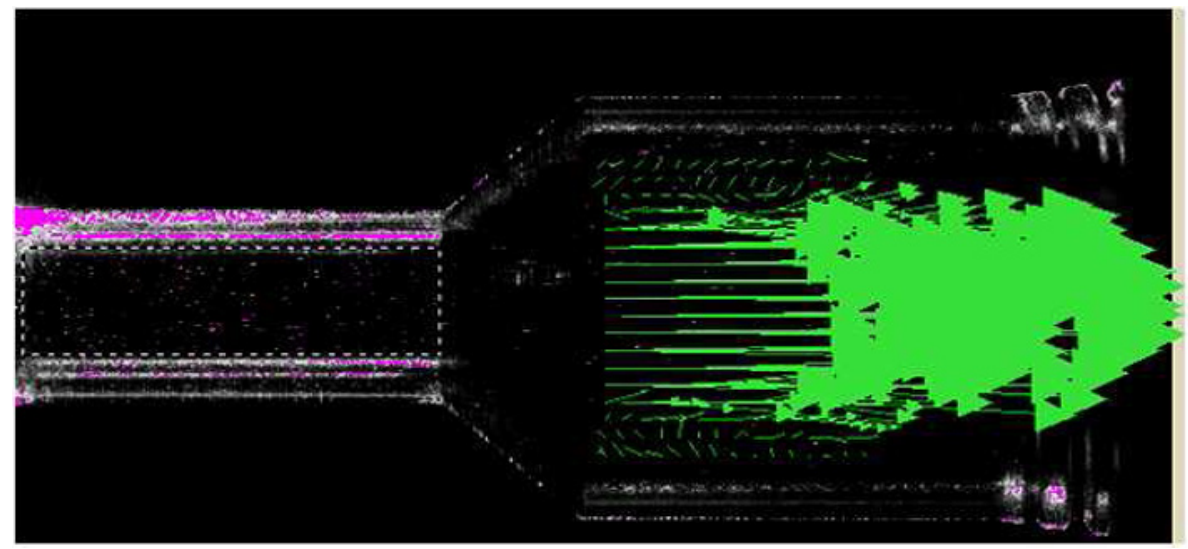

Fig. 11. Downstream velocity flow field obtained by PIV (pink color=reflections) 
Unfortunately, as can be seen in figure 11, while approximately on two thirds of the upstream cross section the velocity profile was found to be similar to that obtained by numerical simulation, the near wall velocity field could not be assessed by using the standard PIV technique. A cause of this is the fact that along a third of the throat and of the expansion diameter there appear reflections and refractions due to the large curvature of the channel and to the differences between the refractive index of the acrylic glass and that of the water. These reflections and refractions manifest as "hidden" areas and/or as image distortions [6]. Due to the poor image quality of these areas, detecting the same particle in two successive images is either impossible or improbable. Applying classical algorithms to determine velocity leads in this case to so-called "spurious vectors" and to the impossibility of determining near-wall velocities.

In conditions when the matching of the refractive indices is difficult to attain due to limitations pertaining to the technological difficulty of creating models of complex configurations from transparent materials, to the constructive aspects of the hydraulic loop components, to elevated costs but also to the problems with the safety of the investigators, a first solution would be to use fluorescent particles (emitting in the red part of the spectrum) and a Fluro $545 \mathrm{~nm}$ optical filter mounted on the camera lens.

On the other hand, to obtain images of sufficient resolution for determining accurately the velocity field, the camera's resolution must be used efficiently, which means focusing on a field of $5 * 5 \mathrm{~mm}$ at most. With the standard lens this was not possible, and focusing was done on almost the entire field. On the basis of the experience accumulated during this experimental programme, we believe that using the telephoto Micro-Nikkor $105 \mathrm{~mm} \mathrm{f/2.8} \mathrm{D} \mathrm{lens} \mathrm{for} \mathrm{close-}$ ups and of one or two extensions ring will enable us to zoom/ focus more close to the wall of the channel.

\section{Conclusions}

For the case we confined ourselves at, the CFD investigation outcomes show the axial symmetry of the flow throughout the successive sudden contraction and expansion as well as the occurrence of two symmetrical recirculation zones. The one that appears downstream of the entrance was confirmed by the PIV results. Unfortunately, with the standard PIV technique currently implemented in our laboratory, the near wall velocity field could not be assessed. Possible solutions to overcome the current technical limitations were identified. Once the necessary materials will be purchased and the imaging system will be upgraded the PIV investigations will be resumed.

\section{Acknowledgment}

This work was supported by the grant of the Romanian National Authority for Scientific Research, CNCS UEFISCDI, project number: PN-II-PD-PCE-2011-30099. A part of this study, referring to the PIV Investigation was partially funded by the Romanian National Authority for Scientific Research, Project Number CNMP 82086 MAACH. Special thanks are due to Stamatios Pothos from TSI Europe for all his help.

\section{REFERENCES}

1. F. Migliavacca, G. Dubini, Biomechanics and modeling in mechanobiology, 3, 235-250 (2005)

2. M.D. Ford, H.N. Nikolov, J.S. Milner, S.P. Lownie, E.M. DeMont, W. Kalata, F. Loth, D.W. Holdsworth, D.A. Steinmam, Journal of Biomechanical Engineering, 2, 15 (2008)

3. A. Ugron, M.I. Farinas, L. Kiss, G. Paal, Exp Fluids, Experiments in Fluids, 52, 37-52, (2011)

4. D. Broboana, T. Muntean, C. Balan, Fluid Mechanics with Fluent, 1, Politechnica Press Publishing House, Bucharest (2005)

5. K.J. Hammad, M.V. Ötügen, E.B. Arik, Experiments in Fluids, 26, 262-272 (1999)

6. R. Budwig, Experiments in Fluids, 17, 350-355 (1994)

7. D.C. Dalal, S.K. Pandt, World Academy of Science, Engineering and Technology, 67, 158-169 (2012)

8. B.F. Armaly, F. Dursts, J.C.F. Pereira, B. Schonung, Journal of Fluid Mechanics, 127, 473496 (1983)

9. W.J. Devenport, E.P. Sutton, Experiments in Fluids 14, 423-432 (1993)

10. http://www.tsi.com/piv-system-2d-measurement 